Machines à voir. Pour une histoire du regard instrumenté, XVII ${ }^{e}-\mathrm{XIX}^{e}$ siècles, anthologie établie, présentée et annotée par Delphine GLEIZES et Denis REYNAUD

\title{
Amélie Calderone
}

\section{(2) OpenEdition}

\section{Journals}

\section{Édition électronique}

URL : http://journals.openedition.org/studifrancesi/15498

DOI : $10.4000 /$ studifrancesi. 15498

ISSN : 2427-5856

\section{Éditeur}

Rosenberg \& Sellier

\section{Édition imprimée}

Date de publication : 1 décembre 2018

Pagination : 497-499

ISSN : 0039-2944

\section{Référence électronique}

Amélie Calderone, "Machines à voir. Pour une histoire du regard instrumenté, $x v I^{e}-x I x^{e}$ siècles, anthologie établie, présentée et annotée par Delphine gLeIzes et Denis reYnAud », Studi Francesi [En ligne], 186 (LXII I III) | 2018, mis en ligne le 01 janvier 2019, consulté le 11 janvier 2021. URL : http:// journals.openedition.org/studifrancesi/15498; DOI : https://doi.org/10.4000/studifrancesi.15498

Ce document a été généré automatiquement le 11 janvier 2021.

\section{(†)

Studi Francesi è distribuita con Licenza Creative Commons Attribuzione - Non commerciale - Non opere derivate 4.0 Internazionale. 


\title{
Machines à voir. Pour une histoire du regard instrumenté, XVII ${ }^{e}$ XIX ${ }^{e}$ siècles, anthologie établie, présentée et annotée par Delphine GLEIZES et Denis REYNAUD
}

\author{
Amélie Calderone
}

\section{RÉFÉRENCE}

Machines à voir. Pour une histoire du regard instrumenté, XVII -XIX sièclesXVII - -XIX $X^{e}$ siècles, anthologie établie, présentée et annotée par Delphine GLEIZES et Denis REYNAUD, Lyon, P.U. Lyon, 2017, «Littérature et idéologies», 402 pp.

1 Machines à voir n'est pas un ouvrage destiné à une lecture suivie in extenso, mais une anthologie à consulter ponctuellement. Encyclopédique, le recueil réussit cependant la gageure d'être rationalisé, raisonné et pensé. Centré sur la France des $\mathrm{XVIII}^{\mathrm{e}}$ et $\mathrm{XIX}^{\mathrm{e}}$ siècles-période présentant une indéniable «unité épistémologique» (p.6) et culturelle-, cet ouvrage se propose «d'interroger l'invention et la diffusion des machines à voir en croisant les apports contemporains de différents domaines artistiques et disciplines scientifiques» (p. 6). Pour ce faire, la perspective choisie est doublement originale: se refusant à une présentation chronologique, les auteurs ont eu l'ambition de contribuer «moins à une histoire des machines qu'à une histoire du regard, ou plus précisément à une histoire du regard instrumenté» (p.6, les auteurs soulignent). Les innombrables «machines», réelles comme fictives, sont au centre des 200 textes, tant scientifiques que littéraires, présentés, annotés, commentés, mais également illustrés, et surtout mis en parallèle et en perspective, au sein de six chapitres analytiques. 
2 Le premier de ceux-ci, «Nouveaux instruments, nouveaux points de vue sur le monde» (pp. 13-123), est le plus fourni: il se présente sous la forme d'un inventaire. Depuis les télescopes jusqu'aux microscopes, en passant par la camera obscura, les lanternes magiques, le daguerréotype ou encore les dioramas, nous est proposée une revue des différentes inventions-y compris celles sans postérité-, qui ont permis soit d'«augmenter les capacités naturelles de l'œil humain» (p. 15), soit de «transform[er] l'expérience sensorielle individuelle en spectacle», soit d'«imiter le mouvement de la vie». Ces objectifs, néanmoins, se voient souvent mêlés en des instruments qui montrent combien le progrès foisonne en des directions multiples et avec des solutions de continuité.

3 Le deuxième, «Spectacles optiques» (pp.125-179), met en lumière les usages spectaculaires de l'optique, particulièrement prégnants au XIX siècle où se sont multipliés tant les spectacles populaires que les mises en scène à vocation pédagogique. Parce que l'image est aussi racoleuse que plus subtilement persuasive, la frontière entre science et charlatanisme est poreuse, ce qui n'empêche pas des usages didactiques, mais également érotiques et politiques, des dispositifs optiques.

4 Une troisième section intitulée «Machines à dérégler les sens: charlatans et philosophes» (pp.181-215), montre combien les divers instruments scientifiques engendrent des pratiques et interprétations irrationnelles, qu'il s'agisse du dévoiement des machines par des escrocs, ou de leur usage heuristique «appel[ant] à un geste philosophique de redressement de l'illusion» (p.183). La sous-partie nommée «Mystification et révélation» (pp. 191-205) se montre particulièrement féconde pour appréhender l'imaginaire du XIX $x^{e}$ siècle, fasciné et façonné par toutes ces inventions aussi sources d'illusion et ce, jusqu'à l'apogée que constitua l'invention des rayons $\mathrm{X}$, forme de merveilleux naturel et scientifique qui a alimenté bien des fantasmes. On notera également le sous-ensemble de textes consacré à la photographie spirite (pp. 207-215), d'autant plus fondamental pour comprendre les rapports entre science et croyance à la fin du siècle qu'ils sont assortis d'une mise en perspective politique: les morceaux recueillis montrent en effet que «la vogue spectaculaire de la photographie spirite a été particulièrement perceptible à la suite de périodes troublées de l'histoire» (p. 207).

5 Le quatrième chapitre, «Visions augmentées» (pp. 217-299), permet de creuser le lien entre voir et savoir, essentiel dans la culture occidentale. Les instruments d'optique ont généré nombre d'extrapolations et ont nourri pléthore d'espoirs illusoires, tel celui de percer le secret des pensées ou de connaître les destinées. L'on comprend mieux la prégnance de certaines figures dans l'imaginaire collectif - songeons, par exemple, à Joseph Balsamo-Cagliostro au XIX ${ }^{e}$ siècle -, «silhouettes inquiétantes marquées par la réprobation, le scandale, la persécution» (p. 221), suscitant fascination et enthousiasme comme répulsion et désespoir. Sont également mis au jour, dans ce chapitre, les modèles politiques fournis par les instruments d'optiques, véritables machines à penser les rapports entre individus. Le panoptique de Bentham en est sans doute un des exemples les plus connus, dont s'inspireront au XIX ${ }^{\mathrm{e}}$ siècle des «architectes d'optique» tels que Ledoux à Arc-et-Senans ou Godin, avec le Familistère de Guise. Les textes ici présentés et commentés ont l'intérêt d'offrir un point de mire nouveau aux problèmes de surveillance des individus auxquels se heurte notre monde contemporain. Il en va de même pour ceux consacrés à toutes les machines destinées à pallier l'absence, invitant tant à la réminiscence et au (res)souvenir intime qu'à la recherche de l'interactivité et 
de la communication à distance - particulièrement sensibles à la fin d'un XIX ${ }^{e}$ siècle qui rêve déjà d'un monde-village et d'une humanité-famille. L'inventivité, à cet égard, s'est également déployée dans le domaine lexical où téléphote (modèle ancestral du téléphone), diaphote et autre télectroscope, ont vainement tenté de concurrencer l'hyperbolique téléchromophotophonotétroscope, ce qui ne fut pas sans alimenter l'ironie qu'ont pu aussi inspirer ces appareils de l'avenir, notamment après les années 1880.

6 L'avant-dernière partie, «Le spectacle de l'histoire» (pp. 301-342), ouvre à une relecture $\mathrm{du}$ renouvellement de l'historiographie caractéristique de la période postrévolutionnaire au prisme de l'œil. L'ensemble des dispositifs optiques permettent en effet, d'une part, d'«interrog[er] l'histoire tout à la fois par les pratiques spectaculaires qu'ils imposent et par l'imaginaire métaphorique qu'ils suscitent» (p.303) et, d'autre part, de questionner le statut de l'observateur. Ils ont ainsi abondamment fourni, au siècle des révolutions, «des spectacles oculaires à même de forger l'identité nationale, voire même de stimuler la fibre nationaliste» (p. 335). Véritables modèles structurants, ils offrent des images propres à illustrer les grands clivages de l'historiographie au XIX ${ }^{\mathrm{e}}$ siècle passée, pour le dire en peu de mots, des ambitions d'un romantisme libéral et républicain désirant fonder la nation et promouvoir le peuple, à une vision critique de cet idéal. L'essor du positivisme, en effet, a nourri un discours national et patriotique. En outre, en tant que dispositifs métaphoriques, ces instruments ont permis de questionner l'effacement ou la présence subjective de l'historien au sein des faits qu'il relate, mais aussi d'engager une réflexion sur «le principe de causalité à l'œuvre dans la production de l'événement» (p. 304).

7 Le sixième et dernier chapitre (pp.343-383), enfin, traite des liens entre optique et psychologie, que le terme réflexion pourrait à lui seul illustrer. L'usage de certaines métaphores est certes stable, quoique leurs significations puissent évoluer. Tel est typiquement le cas de la chambre noire, figurant volontiers les enjeux de la perception et de la connaissance, d'abord annexée aux domaines sensualistes et empiristes, avant de se voir captée par la doctrine spiritualiste deux siècles plus tard. Mais alors qu'au XIX ${ }^{e}$ siècle l'étude de la psyché humaine passe du champ de la morale à celui de la psychologie-discipline alors émergente et discutée-, l'ensemble du paradigme optique se verra remis en cause. Métaphores de la mémoire comme lieu de stockage et comme processus d'inscription, modèles de fonctionnement du cerveau et des visions fantasmagoriques qu'il peut produire, ou encore illustrations de l'activité complexe de la pensée, la photographie, la lanterne magique ou encore le kaléidoscope sont autant d'instruments propres à figurer et penser les mécanismes la psyché humaine, mais également à creuser les états limites du sujet.

Delphine Gleizes et Denis Reynaud ont réussi le difficile pari de parvenir à sélectionner, classer, mettre en perspective, commenter avec concision et pertinence; à rationaliser et rendre lisible, en somme, une matière a priori infinie, foisonnante et hétérogène. Machines à voir est, à cet égard, une anthologie complète, non pas parce qu'elle se veut exhaustive - les auteurs se sont gardés de cette illusoire tentation -, mais parce qu'elle parvient à circonscrire, dans une perspective transdisciplinaire, l'ensemble des enjeux épistémiques, socio-culturels, politiques, littéraires, historiques ou encore ontologiques du champ chronologique étudié. Cette archéologie des représentations, dont la consultation est rendue agréable et aisée par la qualité esthétique de sa présentation, ainsi que par la richesse des illustrations et des index (personarum et rerum) qui 
l'accompagnent, s'imposera comme un outil indispensable aux universitaires travaillant sur les périodes moderne et contemporaine, mais également comme un livre susceptible de séduire les amateurs d'insolite et autres simples curieux. Savantes mais parfaitement intelligibles, ces Machines à voir ont pour qualité principale d'offrir à quiconque les lira une vigoureuse matière à penser. 